\title{
Erratum: ABGC Diplomates Certified in 2002-2003
}

\author{
Anne Greb ${ }^{1,2}$
}

The American Board of Genetic Counseling would like to congratulate the following individuals who achieved certification in 2002-2003. These names were not included in the previously published listing of diplomates. We apologize for this error.

Anderson (Hobson), Alison

Anido, Aimee

Blanco, Amie

Bland, Mary

Conlon, Wendy

Delgado, Julia

Dugan, Rhonda

Genovese, Renee

Hinkson (Verbaan), Deborah

Roche, Frances

Rosen, Ami

Teitelbaum, Ronnie

Wahl, Erica

\footnotetext{
${ }^{1}$ Wayne State University School of Medicine, Detroit, Michigan.

${ }^{2}$ Correspondence should be directed to Anne Greb, Wayne State University School of Medicine, 540 East Canfield, 3216 Scott Hall, Detroit, Michigan 48201; e-mail: agreb@genetics.wayne.edu.
} 